\title{
Ouvrir les mots, ouvrir les morts : dialectiques celaniennes dans Sous votre nom (2015)
}

Plusieurs aspects thématiques et stylistiques colorent Sous votre nom. Tout d'abord, commémorer les morts demeure un enjeu principal. Lenteur et frầcheur se conjuguent lorsque le rite qu'est le poème les accueille. Lénigme et la polysémie des mots poétiques nous permettent de rester en leur présence, d'en raviver le souvenir, de les nommer indirectement et en douceur pour que « s'inverse / le deuil » ( $S N$ 148), pour qu'ils soient perceptibles dans le poème : « Toutes les morts / que nous avions / écrites furent / ouvertes » (53). En reprenant certains mots, rythmes et images celaniens, puis en en trouvant d'autres qui y répondent, Tellermann évoque le vide laissé par la Shoah et suggère un avenir où partager la souffrance peut nous soulager. En tant que femme poète pleurant Celan, Tellermann renverse le mythe d'Orphée au niveau du genre. Celan devient son Eurydice, « sœur » métaphorique $\left(5^{\circ}, 5^{2}, 6 \circ, 92,112,168\right)^{1}$ avec qui elle se lie à des « racines » langagières et sociologiques $\left(5^{\circ}, 5^{2}, 60 ; c f .30,113,120\right)$, c'est-à-dire au langage lui-même comme moteur du devenir et aux morts dont les racines sont dans le ciel. Tellermann redécouvre les absents en tant que communauté et nous annonce l'espoir sinon d'un « Dieu » (112), du moins de lointains pleins de « ferveur » $\left(58,6 \circ, 13^{2}\right)$, de l'éclat et de l'« épaisseur » (206) de nouveaux « récit[s] » (202) que les membres d'un « peuple-sépale » $(168)^{2}$ pourront se raconter.

Sous votre nom s'inscrit donc dans une continuité. Telle une romancière revenant aux thèmes qui la hantent, Tellermann prolonge sa descente dans l'inconscient, explorant musicalement les traces de celui-ci pour mieux comprendre la subjectivité et l'identité, la vie intérieure et l'habiter poétique. Les trois séquences sans titre, d'à peu près quatre-vingts pages chacune, reprennent, dans une « inflexion [...] intimiste », sa méditation de longue date sur « l'érosion des règnes et le pouvoir de la nomination ». ${ }^{3}$ De Contre l'épisode,

\footnotetext{
1 À propos de sœurs métaphoriques, cf. Paul Celan, Choix de poèmes, op. cit., « Je suis seul », [Pavot et Mémoire], p. 67 ; «Greffé sur l'œil », [De seuil en seuil], p. 91 ; «Strette », [Grille de parole], p. 155 ; et « Chymique» [La Rose de Personne], p. 183. Voir aussi « L'écluse », La Rose de Personne, op. cit., p. 31.

2 À propos d'un « sépale », cf. Paul Celan, «Strette », [Grille de parole], Choix de poèmes, op. cit., p. 161.

3 Note de l'éditeur, citée par Angèle Paoli, «Esther Tellermann, Sous votre nom », Terres de femmes, 18 oct. 2015 , < terresdefemmes.blogs.com>.
}

(C) AARON PREVOTS, 2022 | DOI:10.1163/9789004499683_007

This is an open access chapter distributed under the terms of the CC BY-NC-ND 4.0 license. 
il faut retenir aussi la symbolique de l'eau et la mise en mouvement de présences humaines. La méditation s'ancre dans la trajectoire du Je et du Tu que soulignent les césures et incises, les allusions et citations, le souffle comme « trame ardente» où s'entrelacent des voix, ${ }^{4}$ y compris celles des disparus dans le mutisme, dont les noms ont été brûlés. ${ }^{5}$ Respirer devient une façon d'accueillir le temps et l'espace, de se plonger à plusieurs dans le cosmos, de chanter le monde afin que le tissu de la réalité sensible reste entier, ses diverses strates toujours perceptibles.

Sous votre nom se situe dans le contemporain tout en ayant l'envergure d'un mythe, d'une épopée s'inspirant d'anciennes traditions orales. Il y a des énoncés elliptiques, des syntagmes troublants ou censés guérir, mais aussi une structure d'ensemble dont la progression nous rassure, un chant qui s'approche des « légendes » $(S N 31,44,146,202,215)$ et de la prière. L'analyse suivante mettra l'accent sur trois axes d'étude : inventer, naviguer, ouvrir. Ainsi saisira-t-on l'essentiel de Sous votre nom, notamment le fait que le poème nous aide à mieux accueillir la mort, même la plus troublante ou indicible, comme autre face d'un monde « double » (110, 115, 119, 149, 162, 171, 183, 197). Se dévoilent ainsi un équilibre, une possibilité de renouveau et une «inquiétude » (11; cf. 86, 92, 122) illuminante qui côtoie l'envie de «naître» $(161,181$; cf. 69, 195). Le livre Contre-parole de John E. Jackson enrichira nos réflexions. ${ }^{6}$ Traducteur, critique, ami de Celan, Jackson explique en mots simples le désir de celui-ci d'entrer en dialogue avec le langage et l'Autre, et ainsi de s'acheminer vers un avenir pleinement intersubjectif. Contre-parole et les écrits de Celan nous aideront à pénétrer l'énigme dans Sous votre nom, par exemple la présence d'« incendies » d'où peuvent sourdre «Hier» (9) et des « musiques / qui pardonnent » (203).

\section{Inventer}

Tellermann s'adresse autant à autrui dans le moment présent du poème, qu'au langage comme matière et comme moyen d'établir des rapports avec l'Autre. Si à l'instar de Celan elle fait ressentir « l'effort singulier de prendre langue $»,{ }^{7}$ elle le fait dans le but de rassurer ce confrère, de lui dire que la parole peut malgré

4 Pierre-Christophe Cathelineau, «Sous votre nom, Esther Tellermann », La Politique après Freud et Lacan, La Revue lacanienne 17 (2016/1), p. 249-50.

5 Cf. Paul Celan, « Chymique », [La Rose de Personne], Choix de poèmes, op. cit., p. 183, p. 348n2.

6 John E. Jackson, Paul Celan : contre-parole et absolu poétique, op. cit.

7 Jean-Michel Maulpoix commente Choix de poèmes de Paul Celan, op. cit., p. 123, l'auteur souligne. 
tout recoudre nos liens, nous unifier aux niveaux sensuel et sensoriel, culturel et historique, là où nous désespérons des institutions ou du passé collectif. En lisant Sous votre nom, il convient de garder en esprit un propos fondateur dans Le Méridien, discours prononcé le 22 octobre 1960 à la réception par Celan du prix Büchner, sur le langage comme acte de parole qui comprend « [le] souffle, c'est-à-dire une direction et un destin ${ }^{8}{ }^{8}$ Selon Jackson, ce que Celan appelle « la contre-parole ${ }^{9}$ nous libère, en contraste avec les fonctions sémantique, communicative ou sociopolitique du langage qui nous malmènent aux pires moments de l'histoire. ${ }^{10}$ Lorsque Tellermann ouvre les mots, elle tâche justement de leur donner « une direction et un destin ». Là où son aîné André du Bouchet - ami proche de Celan, collègue et traducteur de celui-ci au journal L'Éphémère pendant les années $196 \mathrm{o}^{11}$ - semble mettre en relief l'espace du dehors comme champ qui libère, le minimalisme de Tellermann en fait de même tout en accentuant légèrement l'espace du dedans. Dans chaque cas de figure, le poète résiste aux normes discursives. Selon Clément Layet, il s'agit de faire « retentir le sens comme un cri» par rapport au «silence coupable qui assourdit toute notre époque $» .^{12}$

La spécificité de Tellermann, c'est la plus grande place laissée dans ce contexte à l'intime. Déjà le contenu infime de vers courts et brisés mais étroitement structurés surgit comme trace élémentaire de ce qui va relier le moi intérieur et le monde extérieur. Comme chez Du Bouchet, méditer le réel intensifie toutes sortes de relations, crée un en-avant poétique par lequel nous nous acheminons avec le monde en l'embrassant du regard. Inventer, c'est trouver les outils qui mettront en place la possibilité de tels liens. Ensuite, «inventer » dans Sous votre nom (30, 44, 55, 95, 102, 157, 202, 218, 228), c'est aussi indiquer la présence d'un ou plusieurs interlocuteurs, afin de remettre, comme Celan avant elle, des poèmes à des destinataires réels ou implicites, vivants ou absents. ${ }^{13}$ C'est prendre soin de la langue avec affection et tendresse, veiller

8 John E. Jackson, Paul Celan : contre-parole et absolu poétique, op. cit., p. 13.

$9 \quad$ Ibid., p. 15.

$10 \quad$ Ibid., p. 14-15.

11 Voir Clément Layet, « "La question du soleil" : André du Bouchet et la question politique », L'Esprit Créateur 55:1 (Spring 2015), p. 99-109 (104-09) ; John E. Jackson in Paul Celan, Poèmes, op. cit., p. 68-70 ; James Petterson, Postwar Figures of L'Éphémère : Yves Bonnefoy, Louis-René des Forêts, Jacques Dupin, André du Bouchet, Lewisburg : Bucknell UP, 200o, p. 184-95; et Emma Wagstaff, André du Bouchet : Poetic Forms of Attention, Leiden : Brill Rodopi, 2020, p. $115^{-16 .}$ cit., p. 108 ; cf. $s N 247$.

13 Certaines éditions donnent le contexte dans lequel Celan a écrit ses textes. Voir notamment Choix de poèmes, op. cit. ; Partie de neige, op. cit. ; Renverse du souffle, op. cit. ; et Die 
sur la parole pour la revivifier, accueillir son ombre et sa lumière, accorder nos voix à sa rumeur. Comme il est question de la revigoration d'une langue toujours affaiblie, seront privilégiés chez Tellermann des images profondes et des motifs insistants, ainsi que des blancs, des césures, des espacements, des frémissements de toutes sortes.

Maintes références dès le départ aux recueils de Celan pourront faire penser à lui comme interlocuteur principal, mais, comme d'habitude, son nom n'est pas prononcé. Il est tout à fait possible que le Nous dont s'imprègne Sous votre nom désigne d'autres poètes de l'après-guerre dans sa mouvance, particulièrement Du Bouchet lorsqu'il est question de voir des «versant[s]» (77), de « boire la lumière » (154) ou d'écouter ce qui « nous / suspend / à l'air » (245). Bâtir un livre autour d'« incendies » (9) peut donc faire penser soit au renouveau, soit à ce motif chez Du Bouchet comme reflet d'un dehors qui luit et respire, soit aux traumas de la guerre peut-être enfouis dans l'inconscient et qui vont se changer en nomination du réel. ${ }^{14}$ Il se peut bien que Tellermann esquisse un portrait composite, où se dessinent principalement les traits de Celan, mais également ceux d'autres bien-aimé(e)s, comme pour offrir à chaque page une rose de deuil celanienne à toutes sortes de disparus, bien connus ou sans nom, nous signalant « ce lieu ouvert » que doit être le poème. ${ }^{15}$

Gedichte : Neue kommentierte Gesamtausgabe in einem Band, hrsg. und kommentiert von Barbara Wiedemann, Frankfurt am Main : Suhrkamp, 2018.

Cf. Clément Layet, « "La question du soleil” : André du Bouchet et la question politique », op. cit., p. 99-100. Tout ce qui touche au «feu » parcourt l'œuvre de Du Bouchet. Celan nous semble être néanmoins le principal interlocuteur dans Sous votre nom. Deux brefs exemples du mot «incendie» comme titre chez Du Bouchet: «Alphabet Incendie» (Openwork: Poetry and Prose, trans. and ed. Paul Auster and Hoyt Rogers, Yale UP, 2014, p. 10 [1951]) et «Incendie » (Air suivi de Défets (1950-1953), Saint Clément : Fata Morgana, 1986, p. 35 [1951]). À propos d'éventuels traumas de la guerre que l'on pourrait cerner chez Du Bouchet, ne serait-ce qu'à peine, voir Layet et les Entretiens d'André du Bouchet avec Alain Veinstein (1979-2000), Strasbourg/Paris : L'Atelier contemporain/Institut national de l'audiovisuel, 2016. Tellermann insiste sur le fait que, pour elle, la « dépersonnalisation » l'emporte sur le trauma, lui permettant d'éviter le narcissisme et de se plaire à « refaire l'histoire d'un peuple » mythique, sans nom, «à chaque fois autre et toujours le même » (Anne Malaprade, «Entretien avec Esther Tellermann », op. cit., p. 169). Quant à d'autres poètes qu'elle apprécie, voir $N N$.

Esther Tellermann et Yves Di Manno, «À Martine Broda (1947-2009), 5 mai 2009, cimetière du Montparnasse », Po\&sie 2009/2-3 (128-29), p. 7-11, <cairn.info>; cf. Paul Celan, La Rose de Personne, op. cit. Broda, une amie proche de Tellermann, était pendant un temps la principale traductrice en France de Celan. En parlant du poème comme lieu où se refonde un sens «chaque fois neuf » (7), il est probable que Tellermann pense en partie à la mouvance celanienne dans laquelle elle s'insère elle-même avec Broda et Du Bouchet. 
Bref, Sous votre nom ajoute à l'extrême contemporain une œuvre chorale où s'entretissent en sourdine plusieurs sortes de voix. ${ }^{16}$ Tellermann fait écho à Celan tout en s'en distinguant de par son souffle relativement plus ample, sa propre manière d'aborder les destinataires qui lui sont précieux et la «jouissance $»^{17}$ que soulève son élan.

Prenons d'abord quelques exemples de l'invention comme motif dans Sous votre nom, avant de considérer la manière dont l'invention se poursuit au moyen des leitmotivs si insistants de la navigation et de l'ouverture. Pour le critique voulant aborder l'invention dans ce recueil, c'est un motif à deux facettes : la dizaine de références à l'invention d'un côté, et, de l'autre, la grande inventivité de Tellermann quant à la création d'une réalité sensible où le lecteur peut se plonger pour apprendre à «pardonn[er]» $(S N$ 9). Une image primordiale est celle du titre : l'idée que l'on peut créer des instants « où / cueillir / les mots de dessous » (218) pour en prendre soin et où permettre aux noms d'autrefois de continuer à avoir un sens, à pénétrer notre vécu et rester là parmi nous. Nous revoilà peut-être en plein mythe d'Orphée, mais il s'agit de cueillir les mots pour les contempler et de parvenir au vaste domaine de la mort lentement, amoureusement. Nous nous occupons des mots autant que des êtres. C'est une langue poétique faite par une sorte de prêtre ou de membre d'un chœur antique pour nommer ceux-ci : «je réinventais / le centre des noms / qui ne furent / prononcés » (55). Cela pourrait se référer à tout peuple, à toute ère, n'était le lecteur qui aura pour sa part des lieux de mémoire qui lui viendront à l'esprit.

Voilà l'habiter poétique tel que Tellermann le conçoit, lié à un « Nous » (216) sur les plans affectif, historique, linguistique et ontologique. Certes, cet acheminement vers l'être et les êtres n'est pas toujours calme : « La bouche invente / depuis le gouffre / les masses d'orage » (30). Or, Sous votre nom traite d'un orage de mots qui va déferler en vagues douces, d'une façon de faire fleurir les mots et les êtres sensuellement, comme si les embruns de la mer surgissaient devant le regard : «J'enfantais des / embruns / ne cessais de vouloir / d'autres fièvres / au lieu de mon nom / j'inventais / l'univers » (157). Si gouffre il y a, c'est celui de l'indicible, mais d'un indicible que l'on peut « étrein[dre] » (10), qui fait partie des fièvres dont chacun de nous a besoin pour avoir les pieds sur terre. Un geste féminin - «enfant[er] » les mots - ouvre des horizons et nous montre un possible, allant bien plus loin que la simple invention.

16 Il ne faudrait pas perdre de vue les vers conversationnels chez les anciens, par exemple les Épitres d'Horace (20-14 avant notre ère ; cf. Épittres, trad. et éd. François Villeneuve, Paris : Belles Lettres, 2018).

17 Pierre-Christophe Cathelineau, «Sous votre nom, Esther Tellermann », op. cit., p. 249. 
Qui plus est, lire de tels vers en prenant en compte Celan et la traduction de Jackson nous rappelle que nous ne nous perdons pas dans un gouffre de mots, dans un abîme sans fond. Bien au contraire, feuilleter les pages d'un livre peut correspondre à nos gestes amoureux, comme dans quelques vers d'Enclos $d u$ temps où Celan s'adresse à une bien-aimée et exprime, au moyen d'allitérations, à la fois le désir charnel et le dévouement à l'Autre en tant que libération : « je t'effeuille pour toujours, / / ton étreinte, ta prière / nous libèrent ».18 Tellermann parvient elle aussi à faire remarquer l'épaisseur du langage comme reflet de l'épaisseur du ressenti. Lorsqu'elle parle d'« étreintes » $(S N 10)$, c'est avec une même inventivité et franchise, au sein de vers qui dessinent un univers affectif tout aussi vaste, où figurent le côté charnel des mots et le fond parfois sensuel de nos relations à l'Autre.

Tellermann fait rimer sémantiquement des «étreintes» avec des «horizons » : «plus avant sont / les herbes lisses / en contrefort des / horizons et des / étreintes » (10). La pleine vie que représentent les « herbes lisses » a à voir avec ces «horizons» et ces «étreintes». La vue et le toucher, le corps tellurique et le corps humain, le domaine des images et celui du désir, s'intriquent. De même, la lettre $[\mathrm{h}]$ dans les mots «herbes » et «horizons » renforce les liens qui se laissent percevoir entre le perceptuel et le charnel. Les paysages sont dessinés par « [u]ne pluie violette » (10), celle de l'encre qui crée, puis qui relie, ces versants du réel. Là où Celan aurait peut-être joué sur le côté masculin du désir, la force virile, signe chez lui du courage et de la résilience nécessaires quand frappe le désastre, Tellermann aligne «lumière» et «brume / entre les reins », l'à-venir et l'insaisissable du désir : « Pour vous / nous froissions / l'arc de lumière / brume / entre les reins » (10). Le langage devient matière soyeuse. S'il s'agit d'inventer «des ouvertures / dans le plomb» (102) ou un « orage rouge / à boire » (103), c'est que, à peu près comme Celan, le corps et le désir font partie intégrante de la nomination, vont de pair avec notre éveil au langage comme à l'Histoire. Les séries de textes nous « ouv[rent] » petit à petit au monde, nous invitant à « boire» tout ce qu'un mot peut receler, à intégrer toutes sortes de sensations et de perceptions à notre façon d'être poétiquement avec autrui. Un jeu subtil de références à Du Bouchet qui lui aussi voulait ouvrir le regard, ou à Celan dont le dire est souvent orageux dans son obsession $\mathrm{du}$ « néant » et des morts qui montent au ciel, ${ }^{19}$ accentue le futur collectif que proposent les différentes séquences du texte.

18 Paul Celan, «Les pôles », [Enclos du temps], in John E. Jackson, Paul Celan : contre-parole et absolu poétique, op. cit., p. 142.

19 Paul Celan, «Mandorle», [La Rose de Personne], Choix de poèmes, op. cit., p. 193: «Le néant $[\ldots]$ continue d'être. [...] Ton œil, c'est au néant qu'il fait face.» Cf. «Psaume», 
Quelques dernières citations concernant l'invention mettront en évidence ces aspects sensuels et intersubjectifs du dire tellermannien. La première page de Sous votre nom signale par exemple une problématique celanienne adaptée, celle d'une «éruption » volcanique ${ }^{20}$ qui a lieu «à la limite / de l'âme », éruption à l'égard d'émotions et de pensées et de leur jaillissement en vers au travers d'« incendies » $\left(S N\right.$ 9) de mots isolés. ${ }^{21}$ L'invention fait sentir la chaleur de cette éruption. Tellermann commémore les disparus, élabore des dispositifs qui leur redonnent la parole et intensifie au fur et à mesure la flamme du souvenir, y compris en coloriant de manière enjouée certaines images. Le dialogue affectueux qui en émane est tantôt très immédiat, tantôt lié au passé : « je vous invente dans les / pliures / j'imprime la / couleur afin qu'elle se / sépare » (95); « je vous invente / afin d'accroître / les surfaces / que couvrent les / ornements. / Là font récit / les seuils qui vous ressemblent» (2O2); « dans les orientations / les motifs des / parties basses / je vous / inventais » (44; cf. 218, 228).

Par ailleurs, on observe une progression illuminante dans les images, dans les divers plis des séquences narratives. Tellermann n'hésite nullement à laisser de côté le volcan annoncé au début, pour mettre l'accent sur d'autres résonances linguistiques ou spatiotemporelles. La polysémie, par exemple du mot « pliure » utilisé pour se référer soit au corps, soit à la fabrication de livres, lui permet de frôler ceux à qui elle s'adresse, de parler d'eux tout en élargissant l'étendue de leurs propos, d'« embrase[r] / la durée » (155) et «les espaces de la / durée » (175), de créer d'autres « seuils » et ainsi continuer à communiquer nos liens au langage et à l'Autre. ${ }^{22}$ En réinventant des « seuils » qui accroissent

[La Rose de Personne], p. 181 : « Un Rien, / voilà ce que nous fûmes, sommes et / resterons, fleurissant: / la Rose de Néant, la / Rose de Personne. / / Avec / le style, lumineux d'âme, / le filet d'étamine, ravage de ciel, / la couronne rouge / du mot pourpre que nous chantions, / au-dessus, ô, au-dessus / de l'épine. »

Cf. « Levée de mots, volcanique », Renverse du souffle, dans John E. Jackson, Paul Celan: contre-parole et absolu poétique, op. cit., p. 129 : « Levée de mots, volcanique, / couverte par la rumeur de mer ».

Cf. «Rapport sur l'été », Grille de parole, in John E. Jackson, Paul Celan: contre-parole et absolu poétique, op. cit., p. 127 : «Vide de pas, / contourné, le tapis de thym. / Une ligne de blanc, posée / en travers de la lande aux bruyères. / Rien porté dans le chablis. / / Nouvelles rencontres de / mots isolés, tels : / éboulis, ajoncs, temps. » Voir aussi «(Je te connais », Renverse du souffle, op. cit., p. 47, à propos de l'attente du mot dont les flammes, en témoignant, feront contrepoids à la folie.

À propos de pliures, voir aussi la traduction suivante de «(Je te connais », Renverse du souffle, dans John E. Jackson, Paul Celan: contre-parole et absolu poétique, op. cit., p. 65-66: «(Je te connais, toi, qui es pliée en deux, / moi, le transpercé, te suis assujetti. / Où flambe une parole, qui témoigne pour tous deux ? / Toi - toute, toute réelle. Moi tout entier folie.) » Quant à la polysémie chez Tellermann, le charnel, le tellurique et le linguistique qui s'entrecroisent, voici d'autres références aux « plis » : « de terre » $(S N$ 126) 
« les surfaces », Tellermann laisse à la jouissance sa part. Une parole chagrinée ou agitée - implicitement de la part de Celan et des auteurs sur lesquels il s'appuie - peut se transformer, par exemple, en « lettre historiée », ornée de scènes à plusieurs personnages et marquée par le mouvement dans ses couleurs, sa taille considérable et son «jambage » (39). Les peines vécues par les gens de « l'Est » $(38)^{23}$ deviennent « légendes » ou « incendie [s] » où « la parole » ne meurt pas (44). Leur «pénitences » et « exils » (44) restent gravés dans notre mémoire, mais le Vous ambigu - qui dépasse le Toi plus solitaire et donc affamé « d'incandescence » (247) - nous oriente vers un ici et maintenant où la vue, l'ouïe et le toucher nous aident à témoigner de la présence des êtres et des choses. Inventer ces « vous », c'est surtout « serr[er] / l'écume » (228) que nous offre ce contact renouvelé avec ceux qui nous ont précédés. Le « vous » au présent, en particulier, les rend vivants, alors que le lexique instaure la sensualité et le partage au sein de ces rapports renouvelés.

\section{Naviguer et ouvrir}

Comment parcourir le passé ? Si les mots nous y amènent, que faire une fois arrivé ? Sous votre nom reprend et modifie le dire celanien en mettant en relief à sa manière une Engführung, mot qui veut dire «le fait de mener quelque chose à travers l'étroitesse », mais qui désigne aussi «la dernière partie d'une fugue dans laquelle deux ou plusieurs voix sont reprises en contrepoint, de telle

ou « de chaque jupe » (116); faisant partie d'une « manière / d'ombre et de plis » (140) ou d'« un peu de peau / repliée » (36); liés aux « cycles» (197) ou aux « quelques mots / qui me plient / gardent ouverte / l'incise » (191). Voir aussi Paul Celan, De seuil en seuil, op. cit. Quant aux poetes d'un «Est » imaginaire où se refonde la parole, cf. les remarques suivantes de Tellermann : « [J]'aime la poésie qui est, comme celle d'Hölderlin, tension vers la nomination du monde » (dans Anne Malaprade, «Entretien avec Esther Tellermann », op. cit., p. 168); « Habiter le poème [...] oblige à accueillir l'Histoire, à sortir de l'autobiographie comme de l'anonymat, à construire un sujet contre la puissance du mythe, à bâtir un nom. Je pense, c'est vrai, à Paul Celan, à Ossip Mandelstam, à Trakl ; je leur prends la nuit, l'étoile, la boue et les soleils » (« Poèmes », L'Inconnue de la sublimation, Figures de la psychanalyse 7 (2002/2), p. 161-66 (162), <cairn.info>, à propos de $G E$ ). Voir aussi $N N$. Sous-jacent à l'Est tellermannien est celui non seulement des pénitences et exils (44), mais aussi des richesses culturelles et civilisationnelles qui ont précédé ceux-ci. À travers la biographie de Celan, par exemple, il est possible d'apprécier l'Est bien au-delà du littéraire ou du romantisme allemand, en pensant aux traditions judaïques relatives au Livre, ainsi qu'aux croyances et pratiques faisant partie de la vie quotidienne au sein de diverses communautés. Cf. quant à ce côté sentimental « CEil sombre de septembre », [Pavot et Mémoire], Choix de poèmes, op. cit., p. 37 : «à la fenêtre d'Est / lui apparaît quand il fait nuit l'oblongue / silhouette voyageuse du sentiment». 
sorte qu'à l'instar d'un canon un thème est repris avant que les autres n'aient été menés à leur terme ». ${ }^{24}$ Tellermann emprunte ces visées poético-musicales à Celan - particulièrement évidentes dans son grand poème "Engführung », en français «Strette »- lorsqu'elle dépeint tout au long de Sous votre nom une traversée du réel où le regard embrasse les deux rives de celui-ci dans une traversée quasi nautique. Au moyen de mots qui font écho à Celan, elle explore le passé pour le rendre plus présent, visible, palpable. Elle aide autrui à accéder à la parole, prière sur laquelle se fonde Sous votre nom.

Ces deux spécificités celaniennes - celle de l'étroit et celle d'une fugue où plusieurs voix sont reprises en contrepoint - s'imbriquent. De même que Celan traverse un réel à la fois étroit et plein d'étendues spatiotemporelles afin de s'ouvrir aux disparus et les resituer dans un langage capable de les écouter et de les faire entendre, de même le Je lyrique dans Sous votre nom « démultipli[e] les espaces » où trouver l'Autre, où « recompos[er] / des seuils et des / navires » ( $S N$ 51) pour voyager avec celui-ci. Aux niveaux visuel et sonore, les vers extrêmement courts et verticaux isolent les mots. L'apparaître est là, dans le temps que dure sur la page cette ouverture si mesurée, puis dans la pensée lorsque le devenir des mots et des blancs dans les vers est médité. Quelque chose de secret et de non-dit doit être remarqué, pris en compte, intégré au parler. Celan, pour sa part, semble décortiquer le langage, le regarder comme de l'intérieur à travers la Grille de parole dans laquelle s'insère le poème «Strette», « désassembl[er] » le vécu pour que monte dans le « gris-jour» de la parole «des traces d'eau souterraine ${ }^{25}$ traces des noms en suspens suite aux affres de l'Histoire. ${ }^{26}$ Dans Sous votre nom, il est question de «s'un[ir] » à ce genre de «soir», d'« embrass[er] / tous les gris » $\left(S N{ }_{51}\right)$, de «creus[er] les passages » mais en y apportant des nuances, en allégeant des fardeaux, en « s'abreuv[ant] / à la source des vivants » (27): «Je suis entrée / dans le cristal pour / t'indiquer l'autre / source » (27).

Cette autre source, jamais nommée, sert de repère. Est-ce l'Autre dans la plénitude de sa présence ? La promesse d'une relation à cet Autre, quand bien même nouée à la mort ? L'imaginaire d'où surgit l'ordre symbolique, un tant soit peu plus réglé et apaisé que ce qui se trouve chez Celan ? Tellermann, pour sa part, se réjouit du fait qu'il y ait un « revers de la voix » (236), même si cet autre «monde » (217) ou «bord » risque de « se dissipe[r] » (175). Comme elle écrit Sous votre nom à distance des événements qui, pour Celan, ont marqué le vingtième siècle, il lui est plus facile d'envisager « les / floraisons de dessous »,

24 John E. Jackson, Paul Celan : contre-parole et absolu poétique, op. cit., p. 75.

25 Paul Celan, «Strette », [Grille de parole], Strette \& autres poèmes, op. cit., p. 25-39 (39).

26 Ibid., « Chymique », [La Rose de Personne ], p. 52-53. 
c'est-à-dire une solitude paradoxalement partagée, où plusieurs «voix » peuvent s'unir, minimiser l'aspect sombre de la « roue » de la vie qui ne cesse de tourner : « je te lisais / comme on se couche / au milieu de la / roue / nous nous comptions / en un » (81). Il lui est plus facile de faire d'autres écrivains des « sœurs », pour se lier « aux racines » de la mort et de la vie $\left(5^{\circ}, 5^{2}, 60\right)$, se donner « des / soirs des / océans » (92), mieux avancer « dans / l'incendie » (77).

La progression de ce recueil renforce l'idée d'une navigation suggérée par ces énoncés, tout en y ajoutant des aspects musicaux grâce à des reprises et à la structure d'ensemble. Il y a navigation dans le sens où celle qui parle constate la présence de la matière marquée par le passé, nomme autrui pour être accompagnée en partant à la découverte en profondeur de cette matière, puis compose avec cet Autre «la musique qui pardonne» (243), « respir[e] » avec lui «le voile » $(248),{ }^{27}$ « for [e] / des couloirs » et y dépose des os «afin qu'adviennent / les sources » (78). Il y a des reprises en contrepoint au niveau non seulement des actions effectuées seul(e) ou à plusieurs, mais aussi de l'enchaînement des trois longues parties. Le dire n'est qu'en apparence étroit. Quand « Hier sourd / des tufs » $(9),{ }^{28}$ resteront, d'autant plus visibles à la fin du recueil, des « étendues / vierges / où essaimer[ont] les pierres » de la parole (248), le «fleuve» que chacun « écriv[ait] / tour à tour» (169). Viendra «le Troisième » $(33,248)$, expression qui représente ici l'équilibre des contraires qui se complètent et la lumière profonde qu'apporte la relation.

Comme nous avons pu le voir, cette relation est faite de «sons venus de l'épaisseur» (206), de rites qui «prolong[ent]»(208) ce que d'autres ont dit auparavant, de contrepoints fréquents. Écrire «avec de l'obscur», mais en variant le ton comme le fait Tellermann, exige du lecteur « une attention nouvelle ou accrue ${ }^{29}$ tout en ouvrant de nouvelles brèches dans le dehors. ${ }^{30}$

27 Cf. Paul Celan, « Lointains », De seuil en seuil, op. cit., p. 33. Le voile à respirer ensemble dont parle Celan est au sens figuré un voile de brumes du chagrin, une étoffe langagière teintée de larmes, de mots dont les scories créent des écueils mais dont le pouvoir reste intact. Voir aussi John E. Jackson, Paul Celan : contre-parole et absolu poétique, op. cit., p. 42-43, à propos du poème «Flocons noirs » (Le Sable des urnes, 1948) et d'un Tüchlein, un petit drap qui «pourrait être aussi un linceul [...] dans lequel [...] s'envelopper pour échapper à la fureur [...] de la violence ». Quant au livre Le Sable des urnes, dont des extraits se trouvent dans Pavot et Mémoire, voir Jackson in Paul Celan, Poèmes, op. cit., p. 32.

Cf. Paul Celan, « Esquisse d'un paysage », Grille de parole, in John E. Jackson, Paul Celan: contre-parole et absolu poétique, op. cit., p. 102 : « Tuf de source / où la lumière grandit pour nous, / devant le souffle».

29 Frédéric Marteau, «Contra-diction. Paul Celan et l'art du contrepoint », op. cit., p. 63.

30 Cf. Jacques Depreux, André du Bouchet ou la parole traversée, Seyssel : Champ Vallon, 1988, p. 63, p. 71 . 
Descendre sous les herbes et les mers ( $S N 54,61)$, c'est ainsi traverser non sans bonheur le temps, se baigner dignement « dans [...] les siècles » (82), changer la fugue celanienne - mot qui renvoie aussi au poème «Fugue de mort $»^{31}$ en dialogue souple et ouvert avec ceux qui ont souffert, sans faire d'accusations. Sur le plan thématique, d'ailleurs, Tellermann infléchit cette notion de « fugue » $(78-80,91)$ vers le rêve et la fable, d'abord en faisant une descente mythique pour découvrir cette « déchirure » (78-79), ensuite en se donnant comme Celan une « bouch [e] à ronces » pour tâcher de la chanter (84), tout en $\mathrm{y}$ « superpos[ant] » $(14,91,168,199)$ un éventail d'autres images, par exemple le leitmotiv insistant de l'ouverture : celle des « surfaces » (37), des « morts » (53), des « perles» (103), du « cercle des univers » (22), des « bouches de dessous » (146), voire de « chacun » parmi les pleurants (170). Le passé et le présent s'entremêlent. Naviguer fait valoir « l'infime » (146) et les renversements, le potentiel des êtres et des choses d'« affleure[r]» (147) à travers l'écrit.

Évoquons enfin un poète majeur qui nous a quitté récemment, Salah Stétié (1929-2020). Pour lui, comme pour Tellermann et Celan, il est tout à fait normal qu'un écrivain crée des points de fuite, où ce qui semble disparaître au loin finit par suggérer des voies nouvelles, désigner des lignes qui partiront dans des sens inattendus. Selon Stétié, « [1] a poésie exprime des images profondes, celles que le poète [...] s'en va quérir, avec ses multiples filets, dans les eaux de l'inconscient ».32 Les lieux et les époques peuvent se confondre. Les images elles-mêmes peuvent provenir de ce puits sans fond que sont l'imaginaire et les traditions d'origine culturelle et religieuse qui depuis toujours nous soutiennent. Le poème nous aide à vivre « de ces vastes structures tutélaires » que sont les mythes et qui nous éclairent. ${ }^{33}$ Le «tissage $»^{34}$ de trames mène aux « intériorités communicantes ». ${ }^{35}$

Tellermann fait également signe vers un sens jamais clos, toujours différé, scellé dans les mots, dans leurs rythmes et regroupements, dans les prises de parole, un sens impossible à cerner définitivement et qui compte surtout dans la mesure où il est partagé avec d'autres, en chemin avec eux. C'est ainsi qu'elle ouvre les mots et les morts, en allant vers eux d'un pas patient et décidé. Elle privilégie le rite, le geste sacré d'une prise de parole on ne peut plus ouverte à l'Autre. Comme Celan, elle le fait avec une grande «économie verbale», ${ }^{36}$

Paul Celan, «Fugue de mort» [1945], Pavot et Mémoire, op. cit., p. 83-89; cf. Choix de poèmes, op. cit., p. 332n1.

32 Salah Stétié, En un lieu de brûlure: OEuvres, Paris : Robert Laffont, 2009, p. XXXII.

33 Ibid., p. XXXII.

34 Ibid., p. Xxxvi, l'auteur souligne.

35 Ibid., p. Xxxv.

36 John E. Jackson, Paul Celan : contre-parole et absolu poétique, op. cit., p. 142.
} 
« imprim[ant] dans le vers le plus ténu l'amplitude de l'univers » et approchant ainsi « l'innommable dont nous sommes constitués ». ${ }^{37}$ Le dialogue qu'elle poursuit - en termes celaniens, « l'amande » identitaire de l'être diasporique qu'il faudrait rouvrir ${ }^{38}$ - s'effectue dans Sous votre nom sur le plan de l'allusion, de l'image, de la syllabe, de la strophe, du pli, de l'incise, des pronoms ambigus dont le poème est pétri, de la structure d'ensemble qui affirme la présence de vastes structures tutélaires liées au mythe et à la musique. Que ses prises de parole soient autoréflexives comme chez Celan augmente finalement leur portée, amplifie leur ouverture, permet aux disparus de se sentir chez eux, « de retour $»{ }^{39}$ Ce que le poème de Tellermann nous aide ainsi à percevoir, ce sont d'autres qui peut-être nous ressemblent, « bras ceints autour de la parole » $(S N$ 87 ), naviguant eux aussi avec chaque mot qui « vint par la nuit » et «voulut luire, voulut luire $» .40$

37 Tellermann dans Anne Malaprade, «Entretien avec Esther Tellermann », op. cit., p. 165.

38 Paul Celan, «Mandorle », Choix de poèmes, op. cit., p. 193 ; cf. $S N 88$.

39 Paul Celan, «Strette», [Grille de parole], Strette \& autres poèmes, op. cit., p. 25-39 (25).

40 Paul Celan, «Strette», [Grille de parole], in John E. Jackson, Paul Celan: contre-parole et absolu poétique, op. cit., p. 92. 